\title{
TisN0M1 Sigmoid Colon Cancer: A Case Report
}

\author{
Kyung Ha Lee, Jin Su Kim, Kwang Sik Cheon, In Sang Song, Dae Young Kang', Ji Yeon Kim \\ Departments of Surgery and ${ }^{1}$ Pathology, Chungnam National University Hospital, Daejoen, Korea
}

Distant metastasis of a colon carcinoma in situ has not yet been reported. We experienced a case of a sigmoid colon carcinoma in situ with common hepatic lymph node metastasis. After the first operation, we diagnosed dual intramucosal adenocarcinomas of the sigmoid colon without any regional lymph node metastasis. After the second operation, a metastatic adenocarcinoma was found in the common hepatic lymph nodes. We suggest that metastasis in cases of a colonic carcinoma in situ is rare, but possible. The parallel progression model of tumors can explain this early metastasis.

\section{Keywords: Colon neoplasms; Adenocarcinoma; Carcinoma in situ; Lymphatic metastasis}

\section{INTRODUCTION}

Approximately $20 \%$ of patient with colorectal cancer have distant metastasis at the time of diagnosis. The earlier the $\mathrm{T}$ stage is, the lower the possibility of distant metastasis. One study reported that $1.9 \%$ of $\mathrm{T} 1$ or $\mathrm{T} 2$ colon cancer patients presented distant metastasis perioperatively, and $3.37 \%$ presented after a median of 40.7 months during the follow-up period [1].

Once malignant cells have invaded through the muscularis mucosae, they are able to metastasize, not only to regional lymph nodes, but also to distant organs. However, distant metastasis of a colon carcinoma in situ has not yet been reported. Herein, we report a case of a patient with a pTisN0M1 tumor.

\section{CASE REPORT}

In January 2011, a 71-year-old man was transferred to our facility for surgical treatment of sigmoid colon cancer. His medical history included hypertension and myocardial infarction with three diseased vessels disease, which had been treated in 2007 with per-

Received: July 11, 2013 - Accepted: December 18, 2013

Correspondence to: Ji Yeon Kim, M.D.

Department of Surgery, Chungnam National University Hospital,

282 Munhwa-ro, Jung-gu, Daejeon 301-721, Korea

Tel: +82-42-280-7175, Fax: +82-42-257-8024

E-mail: jkim@cnu.ac.kr

(C) 2014 The Korean Society of Coloproctology

This is an open-access article distributed under the terms of the Creative Commons Attribution NonCommercial License (http://creativecommons.org/licenses/by-nc/3.0) which permits unrestricted noncommercial use, distribution, and reproduction in any medium, provided the original work is properly cited. cutaneous cardiac intervention using a stent. He was taking aspirin, clopidogrel, a beta blocker, angiotensin receptor antagonist, and cholesterol absorption inhibitor. No abnormal finding was found in any preoperative study. The results of the laboratory study, including complete blood count, blood chemistry, coagulation profile and urinalysis, were within normal limits. The level of carcinoembryonic antigen (CEA) and carbohydrate antigen 19-9 were normal as $3.21 \mathrm{ng} / \mathrm{mL}$ and $11.26 \mathrm{U} / \mathrm{mL}$, respectively. In the abdominopelvic computed tomography, one metal clip and a 1-cm polypoid mass was seen in the sigmoid colon (Fig. 1). There was no enlarged peritumoral lymph node, but a $1.3-\mathrm{cm}$ diameter common hepatic lymph node enlargement was found. We considered it most likely to be benign rather than metastatic because primary tumors are considered to be early lesions. On colonoscopy, a one-point $2-\mathrm{cm}$ diameter fungating mass with central ulceration and a $2-\mathrm{cm}$ diameter multilobulated mass were found at $18 \mathrm{~cm}$ and $15 \mathrm{~cm}$, respectively, from the anal verge (Fig. 2). Endoscopy and chest computed tomography (CT) also had no remarkable findings.

The patient underwent a laparoscopic anterior resection (Fig. 3) and was discharged without complications. The pathology report revealed that the lesions in the sigmoid colon were two intramucosal adenocarcinomas with moderated differentiation that had invaded the lamina propria (Fig. 4). The distal and proximal resection margins were clear, and no lymphatic, vascular, or perineural invasion was found. Nine lymph nodes were retrieved, and no lymph node contained malignant cells.

A regular follow-up study was scheduled. Four months after the operation, chest radiography and CEA were normal; however, the common hepatic lymph node had enlarged to $3.4 \mathrm{~cm}$, and multi- 


\section{Coloproctology
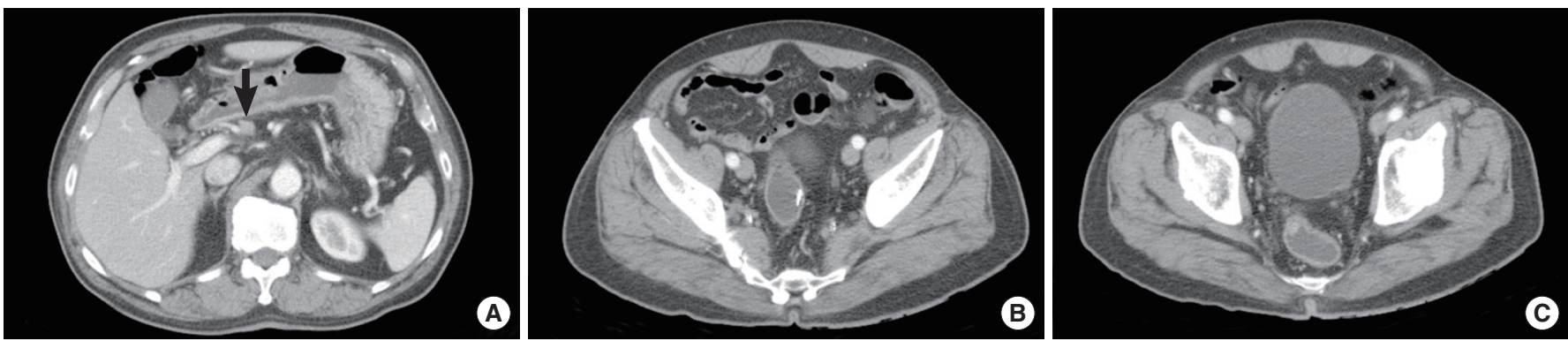

Fig. 1. (A) On initial computed tomography (CT), the common hepatic lymph node (arrow) was enlarged to $1.3 \mathrm{~cm}$, which was considered to be a benign lymph node enlargement upon initial diagnosis. (B) On initial CT, a metal clip is seen in the sigmoid colon. (C) On initial CT, a polypoid mass is seen in the sigmoid colon.
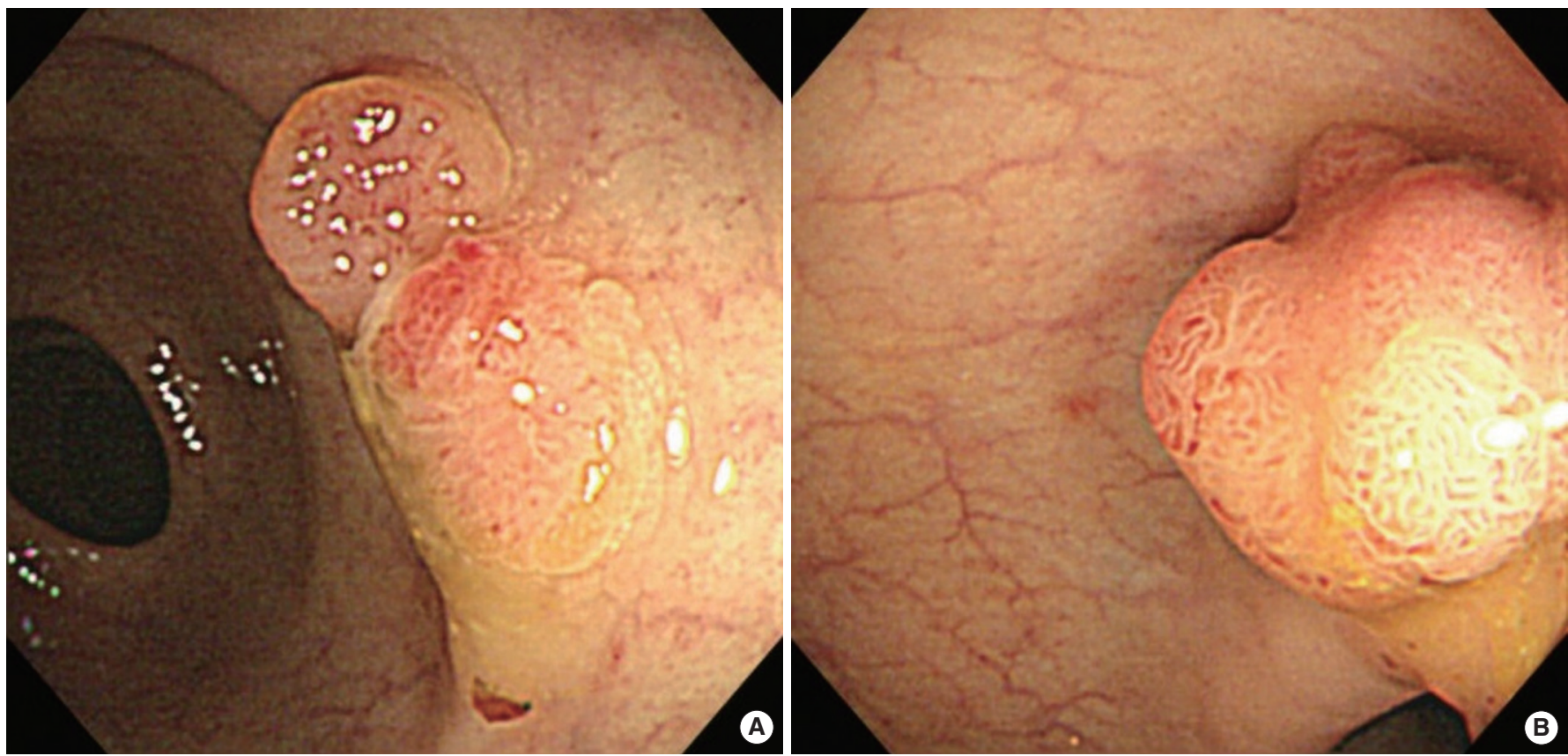

Fig. 2. (A) On initial colonoscopy, a mass is seen at a distance of $18 \mathrm{~cm}$ from the anal verge. (B) On initial colonoscopy, a mass is seen at a distance of $15 \mathrm{~cm}$ from the anal verge.

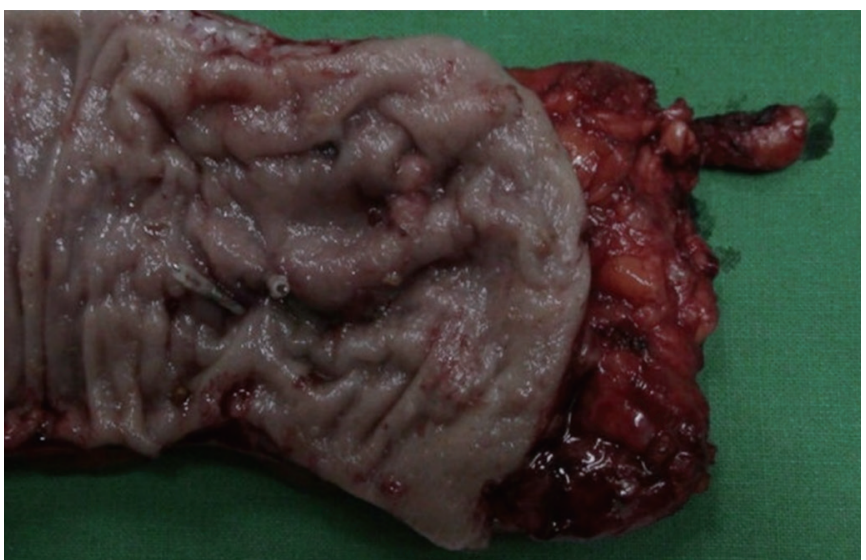

Fig. 3. In the specimen, two adenocarcinomas were found in situ. ple paraaortic lymph nodes were noted on CT (Fig. 5). Diagnostic exploration was recommended, but was denied by the patient due to economic problems. Eight months after the operation, the common hepatic lymph node had enlarged to $6.6 \mathrm{~cm}$, and the sizes of the multiple paraaortic lymph nodes had increased on CT (Fig. 6). Positron emission tomography-CT was performed (Fig. 7). The common hepatic lymph node was considered to be a metastatic lesion; however, there was no uptake of glucose to the paraaortic lymph nodes. An ultrasonography-guided percutaneous biopsy of the common hepatic lymph node was performed, and a metastatic colon adenocarcinoma was diagnosed by a pathologist. A dissection of the common hepatic lymph node was performed by a hepatobiliary surgeon, and the patient recovered without any complications.

The pathology report revealed a metastatic adenocarcinoma 

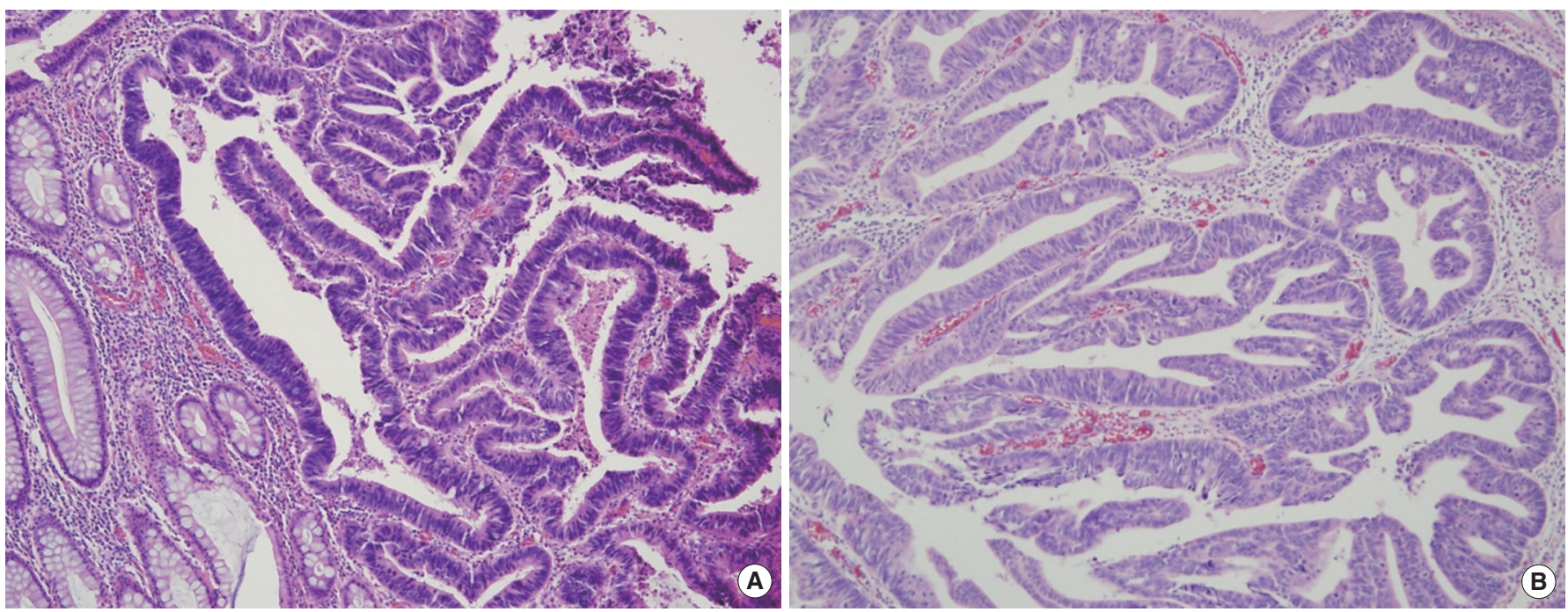

Fig. 4. (A) Pathologic finding shows an intramucosal adenocarcinoma is observed at a distance of $18 \mathrm{~cm}$ from the anal verge (H\&E, $\times 100$ ). (B) Pathologic finding shows an intramucosal adenocarcinoma arising from a tubulovillous adenoma is observed at a distance of $15 \mathrm{~cm}$ from the anal verge $(\mathrm{H} \& \mathrm{E}, \times 100)$.

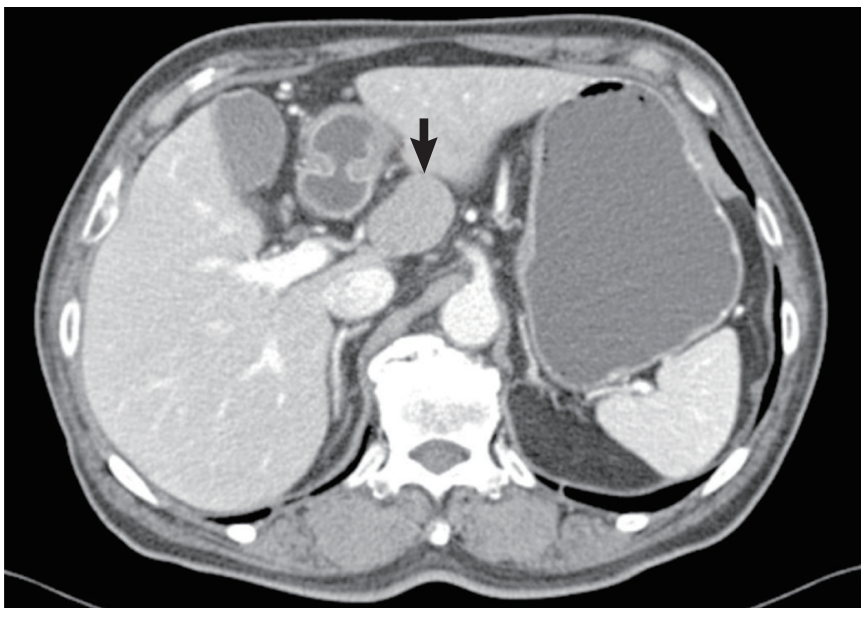

Fig. 5. Computed tomography four months after the operation showed that the size of the enlarged common hepatic lymph node (arrow) had increased to $3.4 \mathrm{~cm}$.

with moderated differentiation, and it showed of positive response for cytokeratin 20 (CK20) and a negative response for cytokeratin 7 (CK7) and antihepatocyte antibody (Fig. 8). To confirm the diagnosis, we checked the responses of the two original masses to CK20 and CK7. The proximal one showed negative responses for both CK20 and CK7, but the distal one showed a positive response for CK20 and a negative response for CK7 (Fig. 9). The patient underwent 12 cycles of FOLFIRI therapy, and during the last follow-up study, which included CT 2 years and 8 months after the surgery, there was no evidence of recurrence.

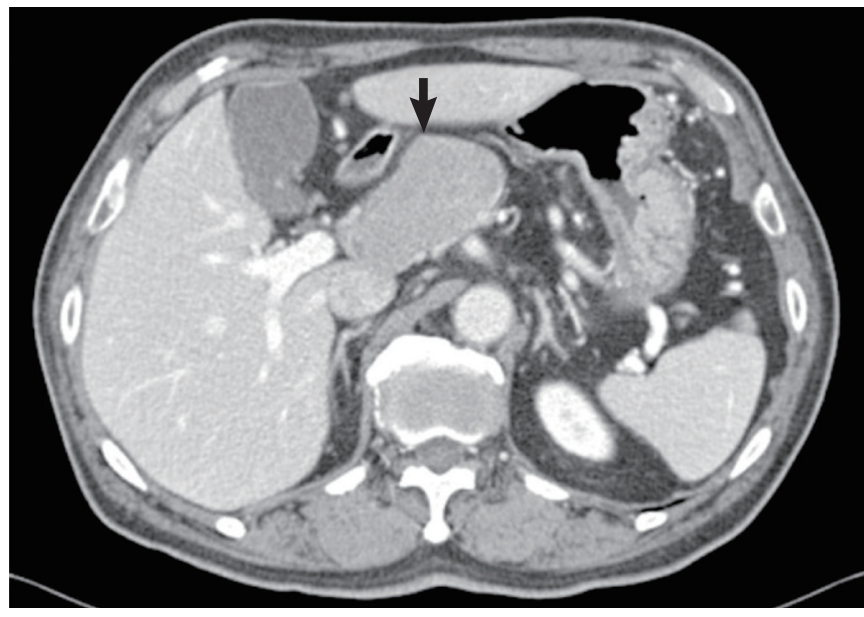

Fig. 6. Computed tomography eight months after the operation showed that the size of the enlarged common hepatic lymph node (arrow) had increased to $6.6 \mathrm{~cm}$.

\section{DISCUSSION}

A colorectal carcinoma in situ includes an intraepithelial and an intramucosal carcinoma. They are defined as malignant cells that are confined to the basement membrane (intraepithelial carcinoma) and that have invaded into the mucosal lamina propria and have extended into, but not through, the muscularis mucosae (intramucosal carcinoma) [2]. A colorectal carcinoma in situ is also referred to as high grade dysplasia. When the tumor is diagnosed as a TisNOM0 tumor, it is classified as stage 0 according to the TNM stage, and the 5 -year survival rate is reported to be $100 \%$. Distant metastasis has been thought to be impossible in a 


\section{Coloproctology \\ TisN0M1 Sigmoid Colon Cancer: A Case Report \\ Kyung Ha Lee, et al.}

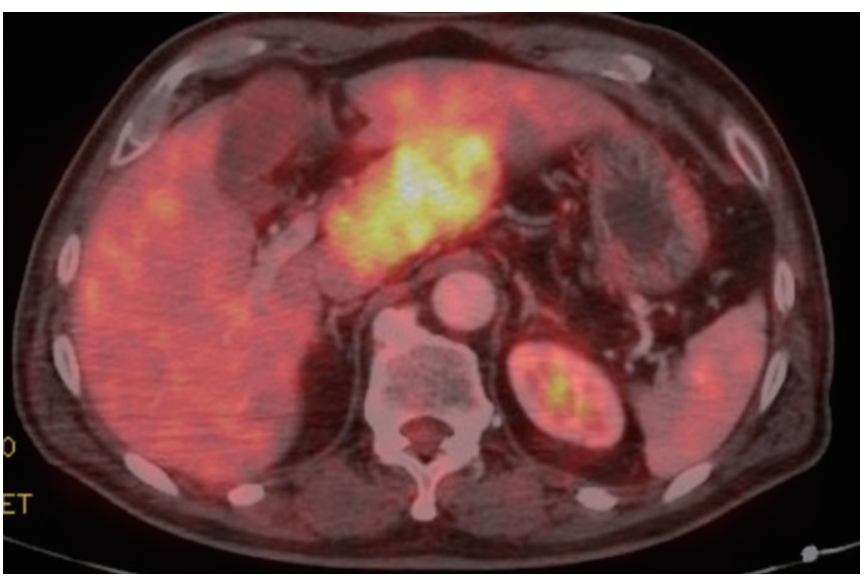

Fig. 7. Positron emission tomography-computed tomography eight months after the operation showed a definite glucose uptake by the enlarged common hepatic lymph node.
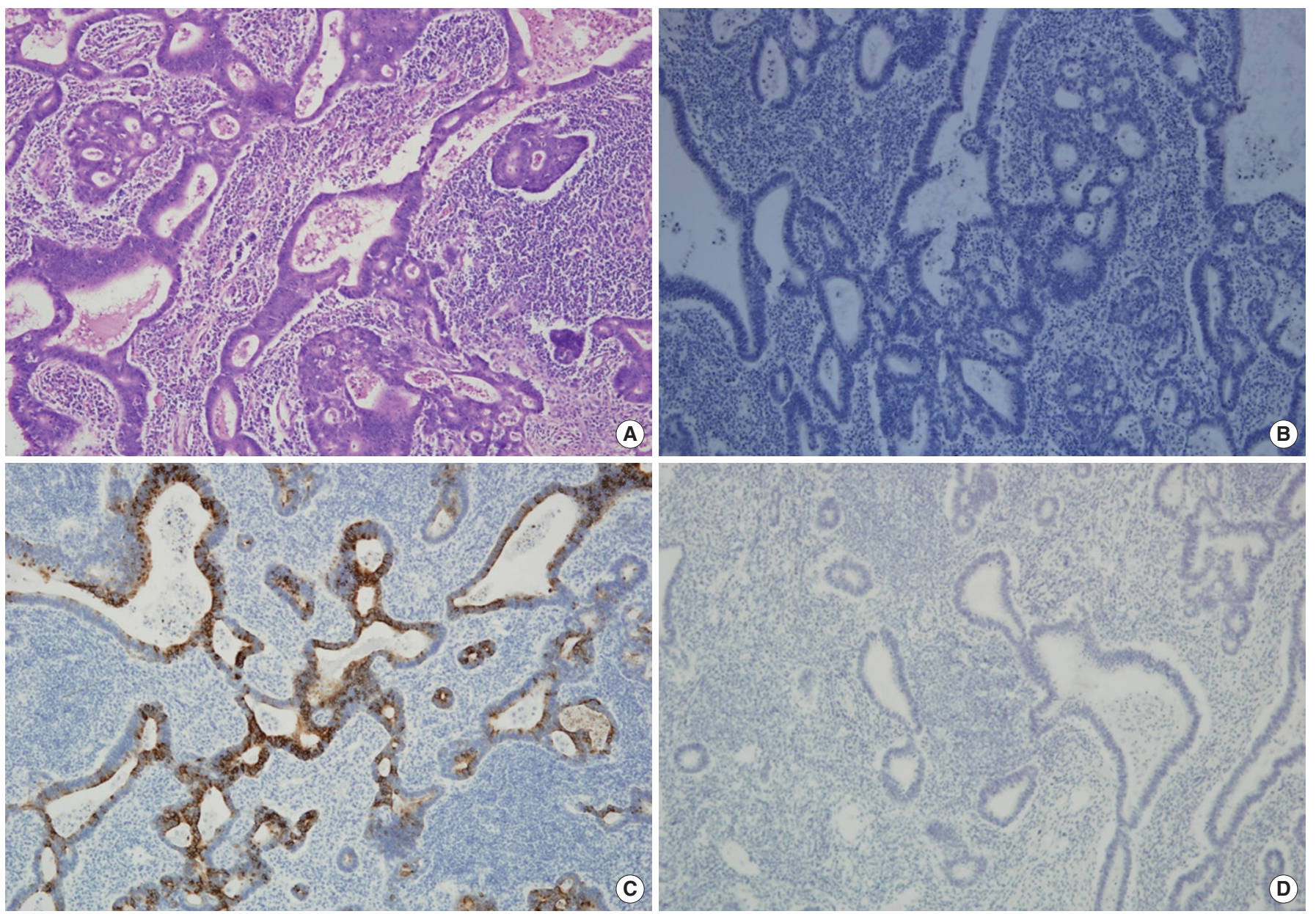

Fig. 8. (A) Pathologic finding shows a metastatic adenocarcinoma of the common hepatic lymph node (H\&E, $\times 100)$. (B) Pathologic finding shows a metastatic adenocarcinoma of the common hepatic lymph node (antihepatocyte antibody negative, $\times 100)$ ) (C) Pathologic finding shows a metastatic adenocarcinoma of the common hepatic lymph node (cytokeratin 20 positive, $\times 100)$. (D) Pathologic finding shows a metastatic adenocarcinoma of the common hepatic lymph node (cytokeratin 7 negative, $\times 100$ ). 

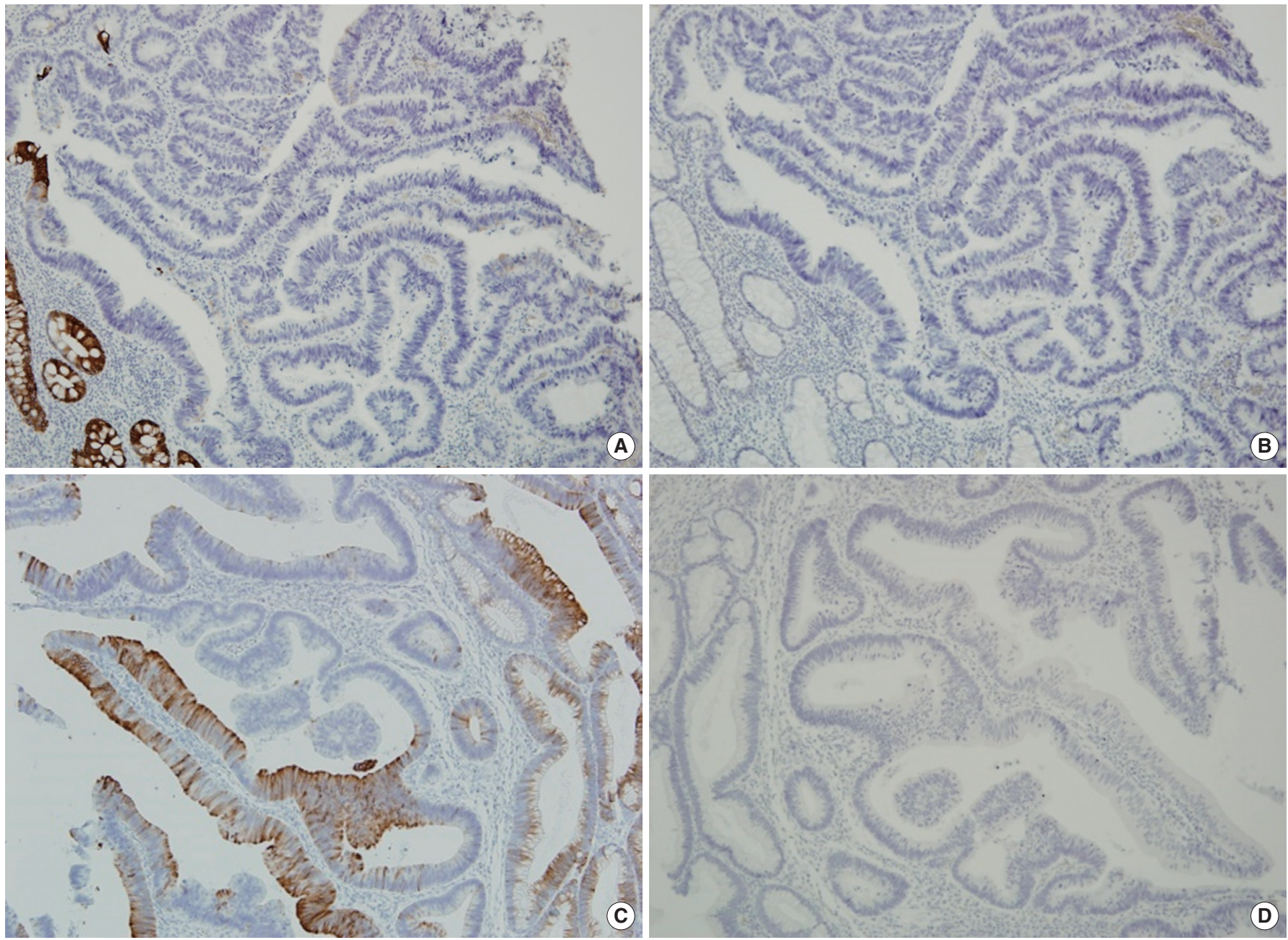

Fig. 9. (A) Pathologic finding shows a proximal intramucosal adenocarcinoma (cytokeratine 20 negative, $\times 100$ ). (B) Pathologic finding shows a proximal intramucosal adenocarcinoma (cytokeratine 7 negative, $\times 100$ ). (C) Pathologic finding shows a distal intramucosal adenocarcinoma (cytokeratine 20 positive, $\times 100$ ). (D) Pathologic finding shows a distal intramucosal adenocarcinoma (cytokeratine 7 negative, $\times 100$ ).

sis is the adenoma-carcinoma sequence, and we cannot deny that most cancers grow according to that principle. However, according to Klein [6], distant metastasis progresses according to two basic models, the linear progression model and the parallel progression model. In the linear progression model, the tumor cell becomes fully malignant in the primary tumor and is then metastasizes. However, in the parallel progression model, tumor cells depart from the primary tumor prior to the acquisition of a full malignant phenotype and undergo a somatic progression and metastatic growth at a distant site. Metastatic founder cells disseminate long before the disease becomes clinically detectable in this model. Our case can be explained by using the second model.

In conclusion, distant metastasis in cases of a colonic carcinoma in situ is rare, but possible. When a distant lesion is found in patients with early colon cancer, including a carcinoma in situ, distant metastasis should not be ruled out.

\section{CONFLICT OF INTEREST}

No potential conflict of interest relevant to this article was reported.

\section{REFERENCES}

1. Fang WL, Chang SC, Lin JK, Wang HS, Yang SH, Jiang JK, et al. Metastatic potential in T1 and T2 colorectal cancer. Hepatogastroenterology 2005;52:1688-91.

2. Compton CC, Greene FL. The staging of colorectal cancer: 2004 and beyond. CA Cancer J Clin 2004;54:295-308.

3. Oien KA, Dennis JL. Diagnostic work-up of carcinoma of unknown primary: from immunohistochemistry to molecular profiling. Ann Oncol 2012;23 Suppl 10:x271-7.

4. Chu P, Wu E, Weiss LM. Cytokeratin 7 and cytokeratin 20 expres- 


\section{Coloproctology}

sion in epithelial neoplasms: a survey of 435 cases. Mod Pathol 2000;13:962-72.

5. Park SJ, Kim JH, Choi CI, Kim DH, Jeon TY, Kim DH. Multiple bone metastases after curative resection of early gastric cancer confined to mucosa without lymph node metastasis. Korean J Clin Oncol 2013;9:66-9.

6. Klein CA. Parallel progression of primary tumours and metastases. Nat Rev Cancer 2009;9:302-12. 